\title{
On analytical transformations for efficiency improvements in the method of finite spheres
}

\author{
Jung-Wuk Hong, Klaus-Jürgen Bathe*
}

Massachusetts Institute of Technology, Cambridge, MA 02139, USA

\begin{abstract}
During the last ten years, various meshless techniques have been developed to solve complex boundary value problems. However, there is still not yet an efficient meshless method available for general linear static analysis. The method of finite spheres is a promising meshless technique with respect to efficiency and applicability [1]. In this paper, improvements of the method of finite spheres are explored based on using analytical transformations prior to the numerical integration.
\end{abstract}

Keywords: Method of finite spheres; Meshfree method; Meshless method

\section{Introduction}

Recently, various meshless techniques such as the smoothed particle hydrodynamics method [2], the diffuse element method [3], and the element free Galerkin method [4] have been developed to solve complex boundary value problems since meshless techniques are very attractive to circumvent mesh generation difficulties. However, still, for the general field of linear static analysis the meshless methods require complex numerical integrations resulting into relatively large solution times. We are developing the method of finite spheres with the aim to reach a meshless method that is in terms of computational effort competitive with traditional finite element methods. For the method of finite spheres to be an effective scheme, improvements in the numerical integration and an efficient auto sphere generation are essential, and we address these issues briefly in this paper.

\section{The Galerkin-based method of finite spheres}

We briefly review in this section the basic formulation of the method of finite spheres.

\footnotetext{
* Corresponding author. Tel.: +1 (617) 253-6645; Fax: +1 (617)
} 253-2275; E-mail: kjb@mit.edu

\subsection{Approximation functions}

Based on the partition of unity requirement, we construct the basis functions. The domain $\Omega \in R^{d}$ is an open bounded domain with $\Gamma$ its boundary as shown in Fig. 1.

Each sphere consists of an open sphere $B_{I}\left(\mathbf{x}_{I}, R_{I}\right)$ and its closure $S_{I}\left(R_{I}\right)$, where $\mathbf{x}_{I}$ is the center of the sphere and $R_{I}$ is its radius. The set of spheres must cover the entire domain, i.e., $\Omega \subset \cup_{I=1}^{N} B_{I}\left(\mathbf{x}_{I}, R_{I}\right)$, and some of them will have non-zero intersections with the boundary; hence those spheres are considered to be boundary spheres.

We define a weighting function $W_{I}\left(s_{I}\right)$, where $s_{I}=$ $\left\|\mathbf{x}-\mathbf{x}_{I}\right\| / R_{I}$. This weighting function has compact support, and we have chosen a quartic spline weighting function of

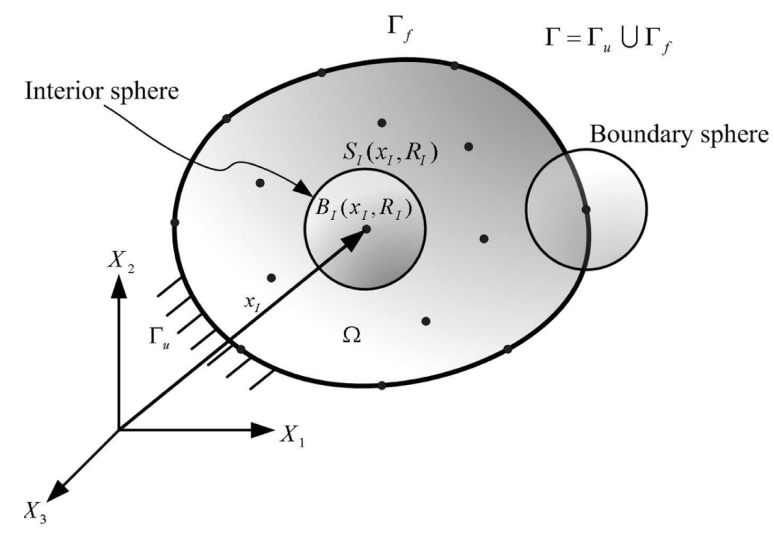

Fig. 1. A schematic of the method of finite spheres. 
the following form:

$W\left(s_{I}\right)=1-6 s_{I}^{2}+8 s_{I}^{3}-3 s_{I}^{4} ; \quad 0 \leq s_{I} \leq 1$.

The weighting functions define the Shepard partition of unity functions

$\rho_{I}(\mathbf{x})=\frac{W_{I}}{\sum_{J=1}^{N} W_{J}}, \quad I=1,2, \ldots, N$.

For generating approximation spaces with higher order consistency, local approximation spaces $V_{I}^{h}=\operatorname{span}_{m \in \mathfrak{F}}\left\{p_{m}(\mathbf{x})\right\}$ are defined at the nodes, where $p_{m}$ is a polynomial function and $\mathfrak{F}$ is an index set. Here $h$ represents the radius of the sphere. We consider in this paper the case $\operatorname{span}_{m \in \mathfrak{F}}\left\{p_{m}(\mathbf{x})\right\}=\{1, x, y\}$ for two-dimensional problems.

The global approximation spaces $V_{h}$ are generated by multiplying the partition of unity function with the local basis polynomial functions,

$V_{h}=\sum_{I=1}^{N} \rho_{I} V_{I}^{h}$

Therefore, any function $v_{h} \in V_{h}$ can be expressed as follows:

$v_{h}=\sum_{I=1}^{N} \sum_{m \in \mathfrak{F}} h_{I m}(\mathbf{x}) \alpha_{I m}$,

where $h_{I m}(\mathbf{x})=\rho_{I}(\mathbf{x}) p_{m}(\mathbf{x})$, and we call $h_{I m}$ the shape function associated with the $m$ th degree of freedom of node $I$, and $\alpha_{I m}$ its coefficient.

\subsection{Displacement-based formulation}

We consider the following variational problem for linear elasticity

Find $u \in H^{1}(\Omega)$ such that

$$
\begin{array}{r}
\int_{\Omega} \epsilon^{T}(v) C \epsilon(u) \mathrm{d} \Omega-\int_{\Gamma_{u}}\left[\epsilon^{T}(v) C N^{T} u+v^{T} N C \epsilon(u)\right] \mathrm{d} \Gamma \\
=\int_{\Omega} v^{T} f^{B} \mathrm{~d} \Omega+\int_{\Gamma_{f}} v^{T} f^{s} \mathrm{~d} \Gamma-\int_{\Gamma_{u}} \epsilon^{T}(v) C N^{T} u^{s} \mathrm{~d} \Gamma \\
\forall v \in H^{1}(\Omega),
\end{array}
$$

where $\epsilon=\left[\begin{array}{lll}\epsilon_{x x} & \epsilon_{y y} & \gamma_{x y}\end{array}\right]^{T}, H^{1}(\Omega)$ is the first order Hilbert space, $u$ is the displacement field, $\epsilon$ is the strain vector, $f^{s}$ is the prescribed surface traction vector on the boundary $\Gamma_{f}, u^{s}$ is the prescribed displacement vector on the boundary $\Gamma_{u}, f^{B}$ is the body force vector, and $N$ is the unit outward normal vector. The approximation for the displacement field can be written as

$u(x, y)=\sum_{J=1}^{N} \sum_{n \in \mathfrak{F}} H_{J n}(x, y) \alpha_{J n}=H(x, y) U$, where $U=\left[\begin{array}{llll}\alpha_{10} & \alpha_{11} & \alpha_{12} & \ldots\end{array}\right]^{T}$ and $\alpha_{J n}=\left[\begin{array}{ll}u^{J n} & v^{J n}\end{array}\right]$. The displacement interpolation matrix and strain-displacement matrix are respectively:

$$
\begin{aligned}
H_{J n}(x, y) & =\left[\begin{array}{cc}
h_{J n}(x, y) & 0 \\
0 & h_{J n}(x, y)
\end{array}\right] ; \\
B_{J n}(x, y) & =\left[\begin{array}{cc}
h_{J n_{,}}(x, y) & 0 \\
0 & h_{J n, y}(x, y) \\
h_{J n_{n},}(x, y) & h_{J n, x}(x, y)
\end{array}\right] .
\end{aligned}
$$

Here, $C$ is the elasticity matrix, in which $E$ and $v$ are Young's modulus and Poisson's ratio, respectively. Finally, the discretized equation for node $I$, degree of freedom $m$, is

$\sum_{J=1}^{N} \sum_{n \in \mathfrak{F}} K_{I m J n} \alpha_{J n}=f_{I m}+\hat{f}_{I m}$,

where

$K_{I m J n}=\int_{\Omega_{I}} B_{I m}^{T} C B_{J n} \mathrm{~d} \Omega$,

$f_{I m}=\int_{\Omega_{I}} H_{I m} f^{B} \mathrm{~d} \Omega$

and $\hat{f}_{I m}$ imposes the displacement/force boundary conditions [5]; for example, for a sphere intersecting $\Gamma_{f}$, we use

$\hat{f}_{I m}=\int_{\Gamma_{f} \cap B_{I}} H_{I m} f^{s} \mathrm{~d} \Gamma$.

In Eqs. (9) and (10), $\Omega_{I}$ is the intersection of $\Omega$ and $B_{I}\left(\mathbf{x}_{I}, R_{I}\right)$.

\section{Improvement of analytical equations for numerical integration}

The expressions to be integrated are rather complex and, clearly, transformations into simpler forms are most desirable. In Eq. (9) we explicitly have:

$K_{I m J n}=\int_{\Omega_{I}}\left[\begin{array}{ll}K_{11} & K_{12} \\ K_{21} & K_{22}\end{array}\right] \mathrm{d} \Omega$,

where the elements of the stiffness matrix are

$K_{11}=C_{11} h_{I m_{, x}} h_{J n_{, x}}+C_{33} h_{I m_{, y}} h_{J n_{, y}}$
$K_{12}=C_{12} h_{I m_{, x}} h_{J n_{, y}}+C_{33} h_{I m_{, y}} h_{J n_{, x}}$
$K_{21}=C_{12} h_{I m_{, y}} h_{J n_{, x}}+C_{33} h_{I m_{, x}} h_{J n_{, y}}$
$K_{22}=C_{11} h_{I m_{, y}} h_{J n_{, y}}+C_{33} h_{I m_{, x}} h_{J n_{, x}}$.

Considering the case $J$ equal to $I$, we can rewrite the equations of the stiffness terms into forms more effective 
for the numerical integration. The integration of the first term in Eq. (13) is

$\int_{\Omega_{I}} h_{I m, x} h_{I n_{, x}} \mathrm{~d} \Omega=\int_{\Omega_{I}} \rho_{I, x}^{2} p_{m} p_{n}+\rho_{I} \rho_{I, x}\left(p_{m, x} p_{n}+p_{m} p_{n, x}\right)+\rho_{I}^{2} p_{m_{, x}} p_{n, x} \mathrm{~d} \Omega$.

The term $\int_{\Omega_{I}} \rho_{I} \rho_{I, x} p_{m, x} p_{n} \mathrm{~d} \Omega$ can be rewritten to obtain

$\int_{\Omega_{I}} \rho_{I} \rho_{I_{x}} p_{m_{x} x} p_{n} \mathrm{~d} \Omega=\int_{\Omega_{I}}\left\{\rho_{I}^{2} p_{m_{x}} p_{n}\right\}_{, x} \mathrm{~d} \Omega-\int_{\Omega_{I}} \rho_{I}\left(\rho_{I} p_{m_{, x}} p_{n}\right)_{, x} \mathrm{~d} \Omega$,

and by the divergence theorem,

$\int_{\Omega_{I}} \rho_{I} \rho_{I, x} p_{m_{, x}} p_{n} \mathrm{~d} \Omega=\frac{1}{2} \int_{\Gamma \cap B_{I}} \rho_{I}^{2} p_{m_{, x}} p_{n} n_{x} \mathrm{~d} S-\frac{1}{2} \int_{\Omega_{I}} \rho_{I}^{2} p_{m_{, x}} p_{n, x} \mathrm{~d} \Omega$.

Similarly, the third term can be transformed

$\int_{\Omega_{I}} \rho_{I} \rho_{I_{x}} p_{m} p_{n, x} \mathrm{~d} \Omega=\frac{1}{2} \int_{\Gamma \cap B_{I}} \rho_{I}^{2} p_{m} p_{n, x} n_{x} \mathrm{~d} S-\frac{1}{2} \int_{\Omega_{I}} \rho_{I}^{2} p_{m, x} p_{n, x} \mathrm{~d} \Omega$.

By substituting Eqs. (19) and (20) into Eq. (17), we obtain

$\int_{\Omega_{I}} h_{I m, x} h_{I n_{x} x} \mathrm{~d} \Omega=\int_{\Omega_{I}} \rho_{I, x}^{2} p_{m} p_{n} \mathrm{~d} \Omega+\frac{1}{2} \int_{\Gamma \cap B_{I}} \rho_{I}^{2} p_{m, x} p_{n} n_{x} \mathrm{~d} S+\frac{1}{2} \int_{\Gamma \cap B_{I}} \rho_{I}^{2} p_{m} p_{n, x} n_{x} \mathrm{~d} S$.

For the other terms in Eqs. (13) to (16) we have

$\int_{\Omega_{I}} h_{I m, x} h_{I n, y} \mathrm{~d} \Omega=\int_{\Omega_{I}} \rho_{I, x} \rho_{I, y} p_{m} p_{n} \mathrm{~d} \Omega+\frac{1}{2} \int_{\Gamma \cap B_{I}} \rho_{I}^{2} p_{m} p_{n, y} n_{x} \mathrm{~d} S+\frac{1}{2} \int_{\Gamma \cap B_{I}} \rho_{I}^{2} p_{m, x} p_{n} n_{y} \mathrm{~d} S$,

$\int_{\Omega_{I}} h_{I m, y} h_{I n_{, x}} \mathrm{~d} \Omega=\int_{\Omega_{I}} \rho_{I, x} \rho_{I, y} p_{m} p_{n} \mathrm{~d} \Omega+\frac{1}{2} \int_{\Gamma \cap B_{I}} \rho_{I}^{2} p_{m} p_{n_{, x}} n_{y} \mathrm{~d} S+\frac{1}{2} \int_{\Gamma \cap B_{I}} \rho_{I}^{2} p_{m, y} p_{n} n_{x} \mathrm{~d} S$,

$\int_{\Omega_{I}} h_{I m, y} h_{I n, y} \mathrm{~d} \Omega=\int_{\Omega_{I}} \rho_{I_{y, y}}^{2} p_{m} p_{n} \mathrm{~d} \Omega+\frac{1}{2} \int_{\Gamma \cap B_{I}} \rho_{I}^{2} p_{m, y} p_{n} n_{y} \mathrm{~d} S+\frac{1}{2} \int_{\Gamma \cap B_{I}} \rho_{I}^{2} p_{m} p_{n, y} n_{y} \mathrm{~d} S$.

We substitute the terms from Eqs. (21) to (24) into Eqs. (13) to (16), and obtain the stiffness terms

$$
\int_{\Omega_{I}} K_{11} \mathrm{~d} \Omega=C_{11}\left\{\int_{\Omega_{I}} \rho_{I_{x}}^{2} p_{m} p_{n} \mathrm{~d} \Omega+\frac{1}{2} \int_{\Gamma \cap B_{I}} \rho_{I}^{2} Q_{1} \mathrm{~d} S+\frac{1}{2} \int_{\Gamma \cap B_{I}} \rho_{I}^{2} Q_{2} \mathrm{~d} S\right\}+C_{33}\left\{\int_{\Omega_{I}} \rho_{I_{y}}^{2} p_{m} p_{n} \mathrm{~d} \Omega+\frac{1}{2} \int_{\Gamma \cap B_{I}} \rho_{I}^{2} Q_{3} \mathrm{~d} S+\frac{1}{2} \int_{\Gamma \cap B_{I}} \rho_{I}^{2} Q_{4} \mathrm{~d} S\right\},
$$

$$
\int_{\Omega_{I}} K_{12} \mathrm{~d} \Omega=C_{12}\left\{\int_{\Omega_{I}} \rho_{I_{I, x}} \rho_{I_{y, y}} p_{m} p_{n} \mathrm{~d} \Omega+\frac{1}{2} \int_{\Gamma \cap B_{I}} \rho_{I}^{2} Q_{5} \mathrm{~d} S+\frac{1}{2} \int_{\Gamma \cap B_{I}} \rho_{I}^{2} Q_{6} \mathrm{~d} S\right\}+C_{33}\left\{\int_{\Omega_{I}} \rho_{I_{,}} \rho_{I_{y}} p_{m} p_{n} \mathrm{~d} \Omega+\frac{1}{2} \int_{\Gamma \cap B_{I}} \rho_{I}^{2} Q_{7} \mathrm{~d} S+\frac{1}{2} \int_{\Gamma \cap B_{I}} \rho_{I}^{2} Q_{8} \mathrm{~d} S\right\},
$$

$$
\int_{\Omega_{I}} K_{21} \mathrm{~d} \Omega=C_{12}\left\{\int_{\Omega_{I}} \rho_{I_{x}} \rho_{I_{y},} p_{m} p_{n} \mathrm{~d} \Omega+\frac{1}{2} \int_{\Gamma \cap B_{I}} \rho_{I}^{2} Q_{7} \mathrm{~d} S+\frac{1}{2} \int_{\Gamma \cap B_{I}} \rho_{I}^{2} Q_{8} \mathrm{~d} S\right\}+C_{33}\left\{\int_{\Omega_{I}} \rho_{I_{x}} \rho_{I_{I,}} p_{m} p_{n} \mathrm{~d} \Omega+\frac{1}{2} \int_{\Gamma \cap B_{I}} \rho_{I}^{2} Q_{5} \mathrm{~d} S+\frac{1}{2} \int \rho_{\Gamma \cap B_{I}} \rho_{I}^{2} Q_{6} \mathrm{~d} S\right\},
$$

$$
\int_{\Omega_{I}} K_{22} \mathrm{~d} \Omega=C_{11}\left\{\int_{\Omega_{I}} \rho_{I_{y}}^{2} p_{m} p_{n} \mathrm{~d} \Omega+\frac{1}{2} \int_{\Gamma \cap B_{I}} \rho_{I}^{2} Q_{3} \mathrm{~d} S+\frac{1}{2} \int_{\Gamma \cap B_{I}} \rho_{I}^{2} Q_{4} \mathrm{~d} S\right\}+C_{33}\left\{\int_{\Omega_{I}} \rho_{I_{x}}^{2} p_{m} p_{n} \mathrm{~d} \Omega+\frac{1}{2} \int_{\Gamma \cap B_{I}} \rho_{I}^{2} Q_{1} \mathrm{~d} S+\frac{1}{2} \int_{\Gamma \cap B_{I}} \rho_{I}^{2} Q_{2} \mathrm{~d} S\right\},
$$


where

$$
\begin{array}{ll}
Q_{1}(x, y)=p_{m_{, x}} p_{n} n_{x}, & Q_{2}(x, y)=p_{m} p_{n_{, x}} n_{x}, \\
Q_{3}(x, y)=p_{m_{, y}} p_{n} n_{y}, & Q_{4}(x, y)=p_{m} p_{n_{y}} n_{y}, \\
Q_{5}(x, y)=p_{m} p_{n_{, y}} n_{x}, & Q_{6}(x, y)=p_{m_{, x}} p_{n} n_{y}, \\
Q_{7}(x, y)=p_{m} p_{n_{,}} n_{y}, & Q_{8}(x, y)=p_{m_{, y}} p_{n} n_{x} .
\end{array}
$$

Using the above transformed expressions for the numerical integration is clearly much more effective than the use of the original expressions. However, ideally similar transformations would also be available for the case $I \neq J$.

\section{Numerical integration}

Since the integrands in Eq. (12) cannot be described by a polynomial function explicitly, numerical integration is unavoidable. We apply a piecewise midpoint rule to integrate the stiffness values as described in [5].

\section{Automatic generation of spheres}

In the method of finite spheres, one of the important tasks is the automatic generation of spheres, which includes the detection of the physical boundary and the determination of the radii for all spheres. There is of course one restriction, namely, that the union of spheres must cover the whole domain.

We adopt a practical way to generate spheres using ADINA input data. The nodal data obtained from ADINA gives the nodes of the spheres. This choice gives us several advantages such as usage of the powerful node generating scheme of ADINA and a convenient result comparison with the exact same node distribution used in a conventional finite element solution. Here it is important to note that while the nodal positions are imported from ADINA, the quality of the positions of the nodes is not critical.

Fig. 2 gives a flow chart showing the use of the ADINA input data and solution process in the program STAP_MFS, which includes the calculation of the radii of the spheres, that is, of the disks in the two-dimensional solutions. The program STAP_MFS has been developed from the STAP program [6] to implement the method of finite spheres.

In the first stage, the ADINA data file is imported to STAP_MFS to generate the LM matrix, i.e., a connectivity matrix for the spheres. This connectivity is based on the nodal positions, the coordinates (x) and radii of all spheres.

The next stage consists of establishing a side table and a boundary table. The information saved in these two tables is used to detect the intersection of spheres with the domain

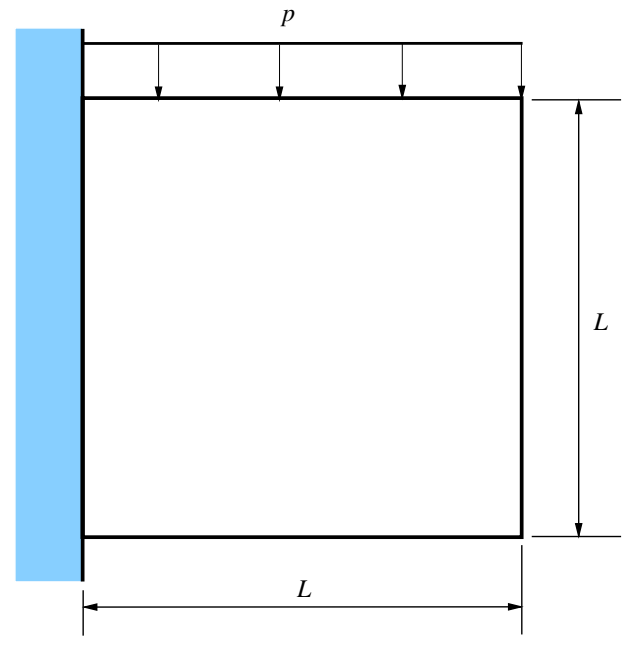

Fig. 3. The cantilever plate for the example case.

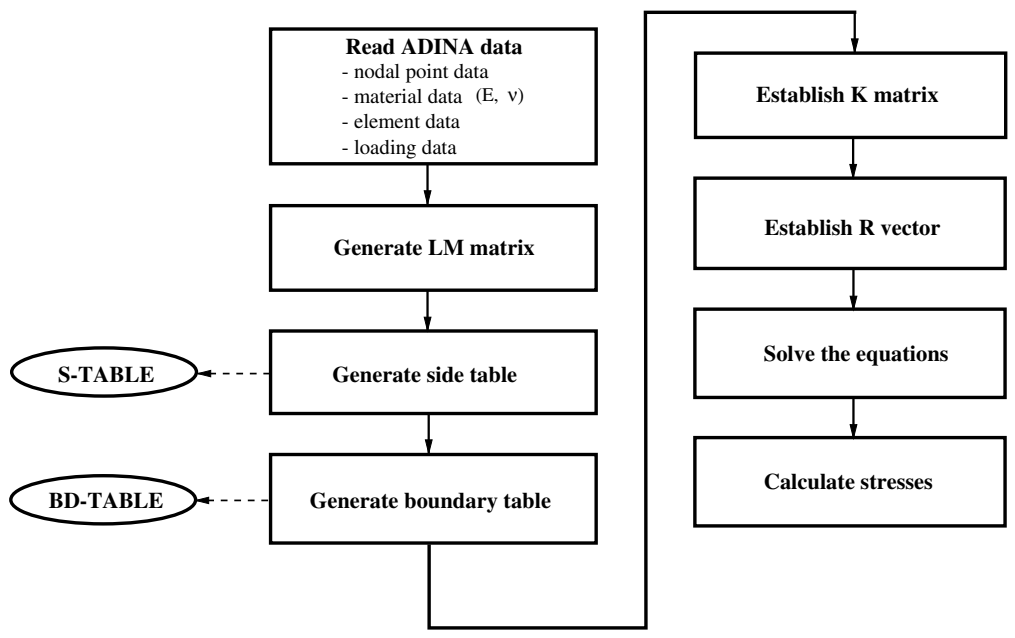

Fig. 2. Flow chart of solution in program STAP_MFS. 


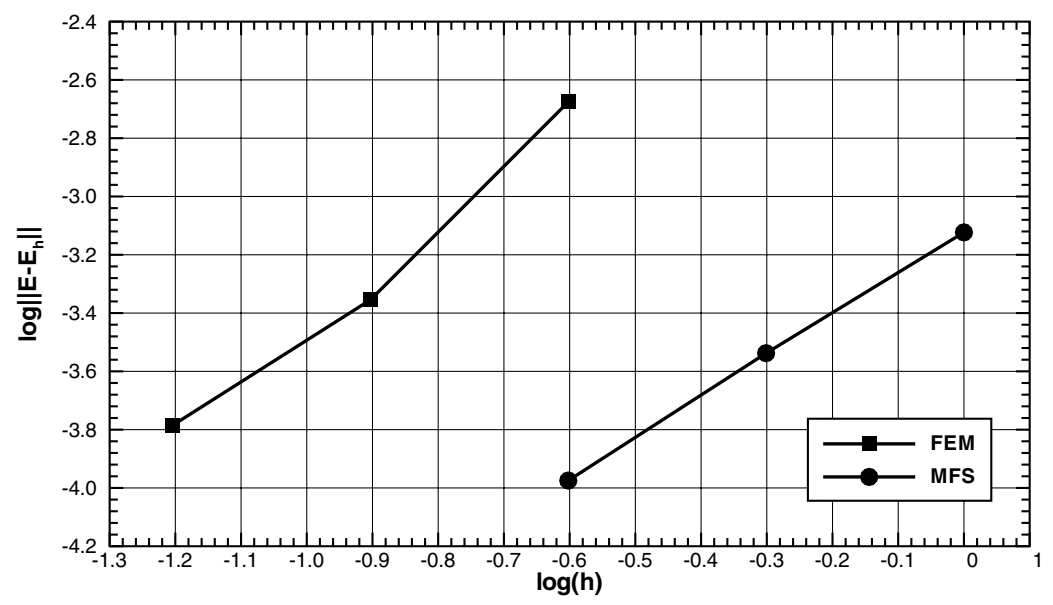

Fig. 4. Convergence comparison of finite element method and method of finite spheres.

boundaries. The process of generating these tables from the ADINA input data is as follows: (1) Connecting the nodes obtained from ADINA, a triangulation is generated. (2) Then searching over all the lines between the nodes of the spheres, we can identify which line is on the boundary of the domain.

We employ spheres of three kinds (inner sphere, contact sphere, boundary sphere). The inner sphere has no contact with the boundary and is inside the domain, the contact sphere has contact with the boundary line and the intersection is a point, and the boundary sphere has its center located on the boundary. The algorithm used to establish the radii of the spheres will be discussed in a future paper.

\section{A numerical result}

The improved formulation was used to solve some typical two-dimensional linear elastic problems. Here we consider a cantilever plate in plane strain conditions (Fig. 3), subjected to uniform pressure loading; we use Young's modulus $E=100$, Poisson's ratio $v=0.3, L=1$, and $p=1$. For the finite element solution, we use 4-node elements in $4 \times 4,8 \times 8$, and $16 \times 16$ element meshes, and for the method of finite spheres $1 \times 1,2 \times 2$, and $4 \times 4$ MFS arrangements were employed.

Fig. 4 shows the results of convergence in strain energy. The figure shows that the method of finite spheres results in the same order of convergence (as expected), but the error is much smaller for a given number of nodal points.

\section{References}

[1] De S, Hong JW, Bathe KJ. On the method of finite spheres in applications: towards the use with ADINA and in a surgical simulator. Comput Mech 2003 (in press).

[2] Monaghan JJ. An introduction to SPH. Comput Phys Commun 1988;48:89-96.

[3] Nayroles B, Touzot G, Villon P. Generalizing the FEM: diffuse approximation and diffuse elements. Comput Mech 1992;10:307-318.

[4] Belytschko T, Lu YY, Gu L. Element free Galerkin methods. Int J Numer Methods Eng 1994;37:229-256.

[5] De S, Bathe KJ. The method of finite spheres with improved numerical integration. Comput Struct 2001;79:2183-2196.

[6] Bathe KJ. Finite Element Procedures. Upper Saddle River, NJ: Prentice Hall, 1996. 